X-ray Radiography and Scattering Diagnosis of Dense Shock-Compressed Matter

S. Le Pape, P. Neumayer, C. Fortmann, T. Doeppner, P. Davis, A. Krichter, O. Landen, S. H. Glenzer

December 23, 2009

Physics of Plasmas 
This document was prepared as an account of work sponsored by an agency of the United States government. Neither the United States government nor Lawrence Livermore National Security, LLC, nor any of their employees makes any warranty, expressed or implied, or assumes any legal liability or responsibility for the accuracy, completeness, or usefulness of any information, apparatus, product, or process disclosed, or represents that its use would not infringe privately owned rights. Reference herein to any specific commercial product, process, or service by trade name, trademark, manufacturer, or otherwise does not necessarily constitute or imply its endorsement, recommendation, or favoring by the United States government or Lawrence Livermore National Security, LLC. The views and opinions of authors expressed herein do not necessarily state or reflect those of the United States government or Lawrence Livermore National Security, LLC, and shall not be used for advertising or product endorsement purposes. 


\title{
X-ray Radiography and Scattering Diagnosis of Dense Shock- Compressed Matter
}

Sebastien Le Pape ${ }^{1}$, Paul Neumayer ${ }^{2}$, Carsten Fortmann ${ }^{3}$, Tilo Doeppner ${ }^{1}$, Paul Davis ${ }^{4}$, Andrea Krichter ${ }^{1}$, Otto Landen ${ }^{1}$ and Siegfried Glenzer ${ }^{1}$

1- Lawrence Livermore National Laboratory, 7000 east avenue, Livermore, CA 94550

2- Gesellschaft fuer Schwerionenforschung (GSI), Darmstadt, Germany

3-Physics Department, University of California Los Angeles, Box 951547, Los

Angeles, California 90095, USA

4- University of California Berkeley, Berkeley, CA 94709, USA

(Dated: November 12, 2009)

\begin{abstract}
Highly coupled Boron plasma has been probed by spectrally resolving an X-ray source scattered by the plasma. Electron density was inferred from the inelastic feature in the collective scattering regime. In addition, the mass density inferred from the noncollective X-ray Thomson scattering has been tested with independent characterization using X-ray radiography in the same drive condition. High-intensity laser produced Kalpha radiation was used as a backlighter for these dynamically compressed plasma experiments providing a high temporal resolution of the measurements. Mass density measurements from both methods are in good agreement. The measurements yield a compression of 1.3 in agreement with detailed radiation-hydrodynamic modeling. From the charge state measured in the non-collective regime and the electron density measured in the collective regime the mass density can then be constrained to $3.15 \pm 0.16$.
\end{abstract}

\section{I- Introduction}

The knowledge of Equation of State (EOS) and related parameters of dense matter is important in several fields of physics. For instance, in astrophysics the star evolution is mainly governed by the thermodynamic properties of matter. Planet cores EOS is fundamental for the knowledge of their internal structure $\left[{ }^{i}\right]$. Optimization of Inertial 
Confinement Fusion (ICF) [ii iii] performance and efficiency will require better understanding of the shell pellet implosion phase and the final core compression, which in turn will require more precise knowledge of the in-flight microballoon material state and the fuel (deuterium) EOS at very high pressures $\left[{ }^{\mathrm{iv}}\right]$ (>100 GPa).

Shock-wave-EOS experiments require that two parameters, usually the shock and fluid velocities, be measured to infer the thermodynamic properties of the material. While a few experiments have used X-ray radiography on low-Z materials to determine both velocities $\left[{ }^{\mathrm{v}}\right]$, most of them $\left[{ }^{\mathrm{vi}}\right.$,iii $]$ rely on the shock velocities measurement (via optical interferometry in transparent media or by observation of shock breakout times on steps of known thickness in optically opaque materials). The latter technique results in indirect EOS determinations through a method known as impedance matching [ ${ }^{\text {viii }}$. Moreover, in measurements based on velocity determination, it is impossible to have a good precision on such an important physical quantity as density due to error amplification inherent to the Rankine Hugoniot relations $\left.{ }^{[\mathrm{ix}}\right]$. The development of direct probing techniques to obtain information on another shock parameter, such as density, would allow precise absolute EOS determinations and represent a real breakthrough in this field. In the first inelastic x-ray scattering measurements on dense plasmas, non-collective backscattering has been performed on isochorically heated solid beryllium targets [ $\left.{ }^{\mathrm{X}}\right]$. These experiments demonstrated accurate temperature measurements from the spectral broadening of the Compton scattering feature that yields the electron velocity distribution. Recent applications of x-ray scattering techniques to probe shocked matter have succeeding in inferring dense matter properties from observation of bound-free $\left[{ }^{\mathrm{xi}}, \mathrm{xi}\right]$ and forward scattering component $\left[{ }^{\text {xiii }}{ }^{x i v}\right]$. Here we present measurement of the mass density of compressed boron using non-collective and collective Thomson x-ray scattering. These measurements are validated by $\mathrm{x}$-ray radiography. Collective scattering provided a robust measurement of the electron density which combined with the charge state of the probed plasma from the non collective scattering constrain with the mass density data 


\section{II- X-ray Thomson scattering}

The experiment was performed on the Titan facility at the Lawrence Livermore National Laboratory ${ }^{11, \mathrm{xv}}$. The experimental setup is presented in figure 1-a. The short pulse beam, $270 \mathrm{~J}$ in $80 \mathrm{ps}$ at $1.053 \mu \mathrm{m}$, is focused by a $\mathrm{f} / 3$ off-axis parabola on a vanadium or copper foil to generate a K $\alpha$ x-ray source. The interaction of an ultrahighintensity laser $\left(>10^{17} \mathrm{~W} / \mathrm{cm}^{2}\right)$ produces $\mathrm{K} \alpha$ fluorescence X-rays from the interaction between hot electrons and cold atoms. The solid density $170 \mu \mathrm{m}$ thick Boron target was shocked compressed by a second high energy beam. A homogeneous intensity distribution over a diameter of $700 \mathrm{um}$ is achieved by use of a continuous phase plate (CPP), providing for a planar shock front to propagate into the target. $400 \mathrm{~J}$ in 5 to $10 \mathrm{~ns}$ at $532 \mathrm{~nm}$ are focused onto the target resulting of about $10^{13} \mathrm{~W} / \mathrm{cm}^{2}$ intensity. Figure 1-b shows a 1D radiation hydrodynamic simulation of the laser-generated pressure in the Boron slab using the code HELIOS at $4 \mathrm{TW} / \mathrm{cm}^{2}$.

At first the electron density was measured in a collective scattering setup. The backlighter was Vanadium, utilizing the V Kalpha line at $4.95 \mathrm{keV}$. Scattering at an angle of 31 degree leading to a scattering vector $\mathrm{k}=1.33 / \AA$ and a scattering parameter $\alpha=1.7$. The scattering parameter is $\alpha=1 / \mathrm{k} \lambda_{\mathrm{s}}=1.7\left[{ }^{\mathrm{xvi}}\right.$ ] where the screening length $\lambda_{\mathrm{TF}}$ is given by the Thomas-Fermi screening length $\lambda_{\mathrm{TF}}=\sqrt{ }\left(2 \varepsilon_{0} \mathrm{E}_{\mathrm{F}} / 3 \mathrm{n}_{\mathrm{e}} \mathrm{e}^{2}\right)=0.43 \AA$ ( where $\mathrm{E}_{\mathrm{F}}$ is the Fermi energy). This approximation is valid for low Te and high density as expected from the hydrodynamic modeling. The scattered light spectrum features two resonances corresponding to scattering off ion acoustic wave and off electron plasma waves, i.e.

plasmons $\left[{ }^{\text {xvii }}\right]$. X-rays scattered by the dense plasma were dispersed by a cylindrically bent $70 \times 25 \mathrm{~mm}$ large graphite crystal (highly-oriented pyrolitic graphite) and focused in a van Hamos setup onto an imaging plate detector (Fujifilm). A $100 \mu \mathrm{m}$ thick gold shield blocked the direct view of the spectrometer crystal to the intense probe source. The gold shield was overcoated with several $\mu \mathrm{m} \mathrm{CH}$ to prevent spectral line from gold emission due to the formation of gold plasma by the intense wings of the drive laser (figure 1-a). According to the hydrodynamic simulation the timing between the backlighter and the long pulse drive is set to 10 ns so that the shock has reached the back of the target and the 
compression is uniform over the slab thickness, which is consistent with the radiography data.

Figure 2 shows the measured scattered spectrum compared to synthetic scattering profiles for different values of the electron density $4.5 \times 10^{23} / \mathrm{cc}, 4 \times 10^{23} / \mathrm{cc}$ and $3.5 \times 10^{23} / \mathrm{cc}$. In this strongly collective and Fermi degenerate scattering regime, the plasmon is undamped in the RPA. To generate a synthetic spectrum, we used the Bohm-Gross dispersion relation $\left[{ }^{\mathrm{xviii}}\right]$, which, at the low temperatures considered here, depends only on the electron density. The spectral weight is inferred via the f-sum rule. The best fit of the plasmon feature is given for an electron density of $4 \times 10^{23} / \mathrm{cc}$.

The non-collective X-ray Thomson scattering measurements have been used to study the shock compression in the same drive conditions. $8.05 \mathrm{KeV}$ (Copper $\mathrm{K} \alpha$ ) photons were scattered at a 63 degree scattering angle, corresponding to a scattering vector $\mathrm{k}=4.3 / \AA$. We are thus in the non-collective scattering regime, $\alpha=0.7$. Figure 3 shows the scattered spectra measured at an angle of 63 degree. The scattered spectrum is fitted using the theoretical form factor of ref $\left[{ }^{\mathrm{xix}}\right]$, it shows that the elastic peak is mostly sensitive to the charge state. Sensitivity with respect to the temperature is weak since we are in the Fermi degenerate regime.

The strength of the elastic scattering component is given by $\mathrm{f}^{2} \mathrm{~S}_{\mathrm{ii}}(\mathrm{k})\left[{ }^{\mathrm{xx}}\right]$. Here $\mathrm{f}$ is the ionic form factor that accounts for the $\mathrm{Zb}$ tightly bound electrons and $\mathrm{S}_{\mathrm{ii}}(\mathrm{k})$ is the ion-ion structure factor. Fig. 3 compares the data to calculation with charge state of 2, 2.25 and 2.5. To calibrate our data, we use the Compton downshifted electron feature, its spectral weight being fixed by the f-sum rule $\left[{ }^{\mathrm{xxi}}\right]$.

Combined with the electron density measured in the collective regime this results in a mass density of $3.15 \pm 0.15 \mathrm{~g} / \mathrm{cc}$, from $\mathrm{rho}=\mathrm{n}_{\mathrm{e}} / \mathrm{Z}$.

\section{III- X-ray radiography}

Point projection $\mathrm{K} \alpha \mathrm{X}$-ray radiography has been used to measure independently from Thomson scattering the mass density of the shock compressed Boron. The short 
pulse laser is focused onto an iron foil to generate a backlighter $\mathrm{K} \alpha$ source at $6.04 \mathrm{KeV}$. This X-ray source provides a temporal resolution of about $100 \mathrm{ps}$ and readily achieves the brightness required for high definition radiography ${ }^{x x i i}$, ${ }^{2 i i i}$. The short duration of the $\mathrm{K} \alpha$ $\mathrm{X}$-ray source reduces the blurring due to the motion of the shock during the interaction of the probe with the shocked target to a value well below the experimental spatial resolution $(18 \mu \mathrm{m})$. For example a shock with a $20 \mathrm{~km} / \mathrm{s}$ velocity travels $1.6 \mu \mathrm{m}$ in $80 \mathrm{ps}$. This source foil was located $2.5 \mathrm{~mm}$ from (figure 4). It produces an $18 \mathrm{x}$ magnified image onto an image plate (Fuji BAS-MS2025) placed at $50 \mathrm{~cm}$ from the shocked target. The resolution was tested using a 1000 lpi (lines per inch) mesh placed at the position of the long pulse laser shocked boron target; the resolution achieved is $18 \mu \mathrm{m}$. A 46-micron Iron filter sandwiched in two $100-\mu \mathrm{m} \mathrm{CH}$ filters to limit the fluorescence of Fe caused by target electrons are placed in front of the Image Plate. A $25 \mu \mathrm{m}$ manganese filter is also used to cut the thermal emission of the iron target.

Shock compression of boron has been studied for different drive condition from 4 $\mathrm{TW} / \mathrm{cm}^{2}$ to $7 \mathrm{TW} / \mathrm{cm}^{2}$. Figure 5 shows a radiograph of a shockwave for a $7 \mathrm{TW} / \mathrm{cm}^{2}(5 \mathrm{~ns})$ drive at $5.6 \mathrm{~ns}$ after the beginning of the long pulse drive. The timing between the short pulse and the long pulse that drives the shockwave has a 150 ps jitter. The shock front is clearly visible due as the opacity increase due to the sudden density augmentation. Figure $6 \mathrm{a}$ and $\mathrm{b}$ show line-outs of the compressed and uncompressed boron for the two different drive conditions (4 and $7 \mathrm{TW} / \mathrm{cm}^{2}$ ) and two time delays at 5.6 and $7 \mathrm{~ns}$ respectively.

The line-outs show the absorption feature as the shock wave propagates in the boron slab, the shock front is observed further into the boron for the longer delay (7 ns). The density in the compressed boron is deduced from the ratio of absorption in the compressed region to the uncompressed region. For a quantitative analysis we have used the Monte-Carlo code ITS $\left[{ }^{\mathrm{xxiv}}\right]$ to model both the $\mathrm{K} \alpha$ and bremstrahlung production as well as the absorption in the Boron.

The contrast between the shocked and unshocked region is strongly dependant on the backlighter spectrum. From the absorption in the step filters placed in the field of view of the Image Plate, we know that the $\mathrm{X}$ ray spectrum feature a high-energy component. We have used ITS to model the $\mathrm{X}$ ray emission and adjusted the hot electron distribution input to match the absorption in the silver step filters and the unshocked boron. 
The Beer-Lambert law gives the absorption in the shocked material:

$$
\frac{I}{I_{0}}=\exp (-\mu \rho L)
$$

Where $\mu$ is the cold opacity, $L$ the length of the shocked region and $\rho$ the mass density.

The shock front is assumed to be planar over the size of the focal spot, i.e $750 \mu \mathrm{m}$. The density in the shocked front for the $7 \mathrm{TW} / \mathrm{cm}^{2}$ is $3.9 \pm 0.6 \mathrm{~g} / \mathrm{cc}$ and $2.9 \pm 0.6 \mathrm{~g} / \mathrm{cc}$ for a 4 $\mathrm{TW} / \mathrm{cm}^{2}$. The main source of error in the density determination is the noise in the lineout.

\section{IV-Conclusion}

We have demonstrated the ability to use non-collective x-ray scattering to measure the density of shock-compressed matter in excess of 1 Mbar. The density inferred from the scattered spectra has been independently measured using X-ray radiography. Using a $4 \mathrm{TW} / \mathrm{cm}^{2}$ laser drive, we have measured a compression of 1.3 in good agreement with radiation hydrodynamic modeling. The mass density was also measured from a combination of the shift of the inelastic feature in the collective scattering regime and the elastic strength in the non-collective scattering regime. From the electron density measured to be $4 \times 1023 / \mathrm{cm} 3$ and charge state $=2.3 \pm 0.16$, the mass density can be constrained to $3.15 \pm 0.2 \mathrm{~g} / \mathrm{cc}$, consistent with the radiography result.

\section{ACKNOWLEDGMENTS}

This work was performed under the auspices of the Department of Energy by Lawrence Livermore National Laboratory under Contract No. DE-AC52-07NA27344. The work and vital assistance of the technical, scientific and administrative staff connected with the 
Jupiter Laser Facility at LLNL are gratefully acknowledged, LDRD 08-ERI-004 by the Alexander von Humboldt Foundation.

\section{References}

${ }^{i}$ D.J. Stevenson, Models of the earth's core, Science 214 (Nov. 6, 1981) 611-619 (Research supported by California Institute of Technology ).

ii Nuckolls, John; Wood, Lowell; Thiessen, Albert; Zimmerman, George, Nature, Volume 239, Issue 5368, pp. 139-142 (1972)

iii M. D. Knudson, M. P. Desjarlais, D. H. Dolan, Phases of Carbon Shock-Wave Exploration of the High-Pressure, , 1822 (2008); 322 Science

${ }^{\text {iv }}$ G.A. Collins, Science 281 (1178) (1998).

${ }^{v}$ R. Cauble, T.S. Perry, D.R. Bach, K.S. Budil, B.A. Hammel, G.W. Collins, D.M. Gold, J. Dunn, P. Celliers, L.B. da Silva, M.E. Foord, R.J. Wallace, R.E. Stewart, N.C. Woolsey, Absolute equation-of-state data in the 10e40 Mbar (1e4 TPa) regime, Physical Review Letters 80 (6) (February 9, 1998) 1248e1251. intense laser interactions with solids, Physical Review Letters 84 (4) (January 24, 2000) 670-673.

${ }^{\text {vi }}$ M. Koenig, B. Faral, J.M. Boudenne, D. Batani, A. Benuzzi, S. Bossi, C. Remond, J.P. Perrine, M. Temporal, S. Atzeni, Relative consistency of equations of state by laser driven shock waves, Physical Review Letters 74 (12) (March 20, 1995) 2260-2263.

vii A. Benuzzi-Mounaix, M. Koenig, G. Huser, B. Faral, D. Batani, E. Henry, M. Tomasini, B. Marchet, T.A. Hall, M. Boustie, Th. de Resseguier, M. Hallouin, F. Guyot, D. Andrault, Th. Charpin, "Absolute equation of state measurements of iron using laser driven shocks", Physics of Plasmas 9 (6) (2002) 2466. Communications 49 (3) (1988) 475-505. 
viii T. R Boehly, J. E Miller, D. D Meyerhofer, J. H Eggert, P. M Celliers, D. G Hicks, G. W Collins, Shock Compression of Condensed Matter, 2007 vol. 955 pp. 19.

${ }^{\text {ix }}$ P. M. Celliers, D. K. Bradley, G. W. Collins, and D. G. Hicks, R.S.I. 75, NUMBER 11 NOVEMBER 2004

${ }^{x}$ Glenzer, S. H.; Gregori, G.; Lee, R. W.; Rogers, F. J.; Pollaine, S. W.; Landen, O. L., Phys. Rev. Lett. 90, 175002 (2003).

${ }^{x i}$ H Sawada, S. P Regan, D. D Meyerhofer, I. V Igumenshchev, V. N Goncharov, T. R Boehly, R Epstein, T. C Sangster, V. A Smalyuk, B Yaakobi, G Gregori, S. H Glenzer, O. L Landen, Physics of Plasmas, 2007 vol. 14 pp. 2703

xii A Ravasio and G Gregori and A Benuzzi-Mounaix and J Daligault and A Delserieys and AY Faenov and B Loupias and N Ozaki and M Rabec Le Gloahec and TA Pikuz and D Riley and M Koenig, PRL 99, 135006 (2007)

xiii E Saiz, G Gregori, D Gericke, J Vorberger, NATURE PHYSICS, 2008 p 7270

xiv Kritcher, Andrea L.; Neumayer, Paul; Castor, John; Döppner, Tilo; Falcone, Roger W.; Landen, Otto L.; Lee, Hae Ja; Lee, Richard W.; Morse, Edward C.; Ng, Andrew; Pollaine, Steve; Price, Dwight; Glenzer, Siegfried H., 69 (2008) 322 Science

${ }^{x v}$ See National Technical Information Service Document No. XXXXX (awaiting assignment), "Titan leads the way in laser-matter science", Science and Technology Review, Jan-Feb 2007, pp4-11. Copies may be ordered from the National Technical Information Service, Springfield VA 22161

${ }^{\text {xvi }}$ Siegfried H. Glenzer and Ronald Redmer, Review of modern physics accepted for publication 2009

${ }^{\text {xvii }}$ S. H. Glenzer et al., PRL 98, 065002 (2007)

xviii D. Bohm and E. P. Gross, Phys. Rev., 75, 1851 (1949) 
${ }^{\text {xix }}$ G. Gregori , A. Ravasio , A. Ho, S.H. Glenzer , S.J. Rose, High Energy Density Physics 3 (2007) 99-108

${ }^{x x}$ Junzo Chihara, J. Phys.: Condens. Matter 12 (2000) 231-247

${ }^{x x i}$ Kraeft et al, Quantum Statistics of Charged Particle Systems, Akademie Verlag Berlin 1986

xxii Park, H.-S.; Izumi, N.; Key, M. H.; Koch, J. A.; Landen, O. L.; Patel, P. K.; Phillips, T. W.; Zhang, B. B., Review of Scientific Instruments, Volume 75, Issue 10, pp. 4048$4050(2004)$

xxiii King, J. A.; Akli, K.; Zhang, B.; Freeman, R. R.; Key, M. H.; Chen, C. D.; Hatchett, S. P.; Koch, J. A.; MacKinnon, A. J.; Patel, P. K.; Snavely, R.; Town, R. P. J.; Borghesi, M.; Romagnani, L.; Zepf, M.; Cowan, T.; Habara, H.; Kodama, R.; Toyama, Y.; Karsch, S.; Lancaster, K.; Murphy, C.; Norreys, P.; Stephens, R.; Stoeckl, C., Applied Physics Letters, Volume 86, Issue 19, id. 191501 (3 pages) (2005).

${ }^{\text {xxiv }}$ J. A. Halbleib, R. P. Kensek, G. D. Valdez, S. M. Seltzer, and M. J. Berger, IEEE Trans. Nucl. Sci. 39, $1025 \square 1992 \square$ 


\section{Figures captions}

Figure 1: a- Schematic of the experimental arrangement b- 1D radiation hydrodynamic calculation from HELIOS showing a pressure above 1Mbar in the boron as a function of time in the boron. The laser comes from the right.

Figure 2: $\mathrm{X}$ ray scattering data for 31 degree scattering angle, a scattering vector $\mathrm{k}=1.3 / \AA$ along with theoretical fit for various electron density $3.5 \times 10^{23} / \mathrm{cc}, 4 \times 10^{23} / \mathrm{cc}$ and 4.5 $\mathrm{x} 10^{23} / \mathrm{cc}$.

Figure 3: $\mathrm{X}$ ray scattering data for a 63 degree scattering angle, a scattering vector $\mathrm{k}=$ 4.3/ $\AA$, along with theoretical fit for various $\mathrm{Z}$. $\mathrm{Te}=0.2 \mathrm{eV}, \mathrm{Z}=2.2$

Figure 4: Schematic of the experimental arrangement

Figure 5: Digitized radiograph of the compressed Boron taken at $5.6 \mathrm{~ns}$ for a $7 \mathrm{TW} / \mathrm{cm}^{2}$ laser drive. The laser comes from the right; the absorption in the shock wave is clearly visible in the Boron slab. On the side of the image the absorption of the step filters is visible.

Figure 6: Lineout along the laser axis of the compressed (dashed blue curve) and uncompressed boron. The decrease in signal indicates absorption feature that is correlated with an increase of the density in the shock wave for (a) $7 \mathrm{TW} / \mathrm{cm}^{2} 5.6 \mathrm{~ns}$ delay (b) for a $4 \mathrm{TW} / \mathrm{cm}^{2}$ drive 7 ns delay. 


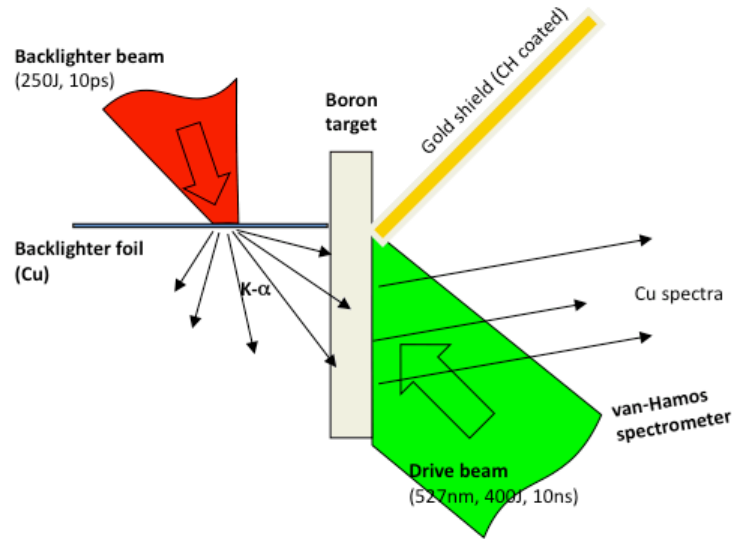

a

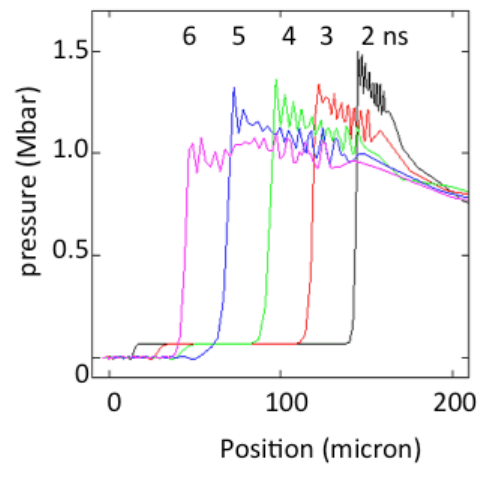

b

\section{figure 1}




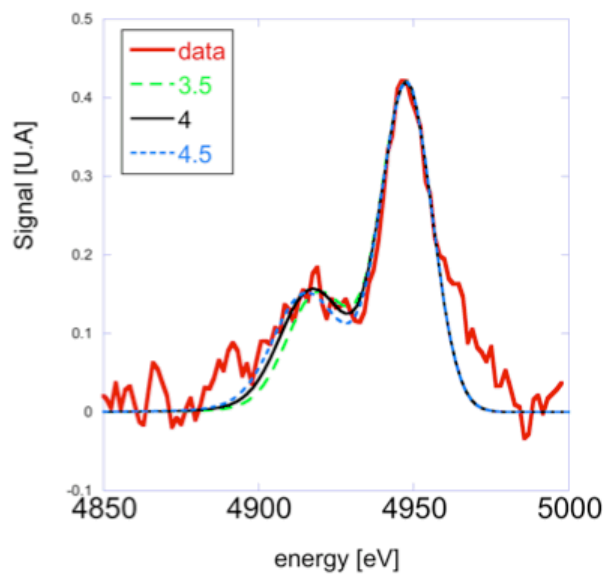

figure 2 


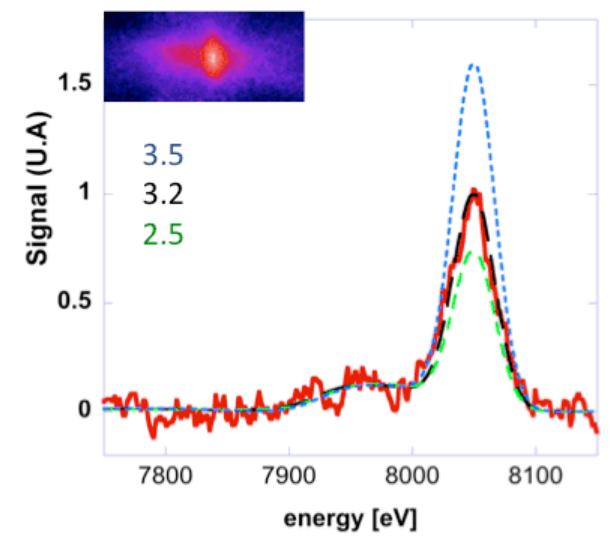

figure 3 


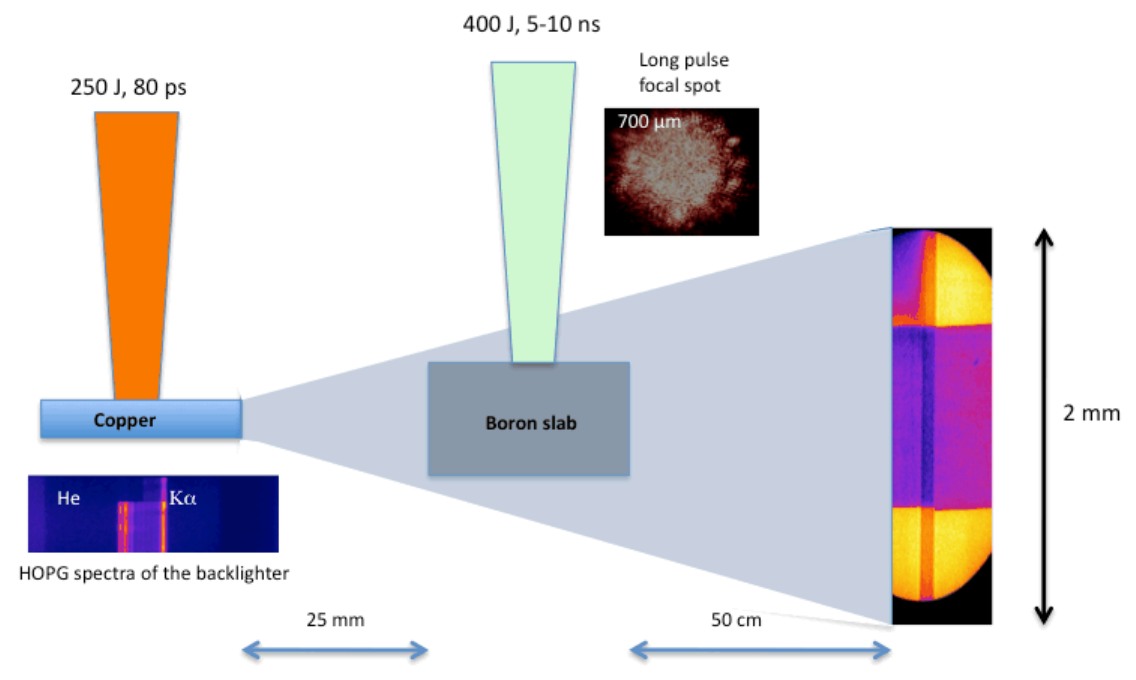

figure 4 


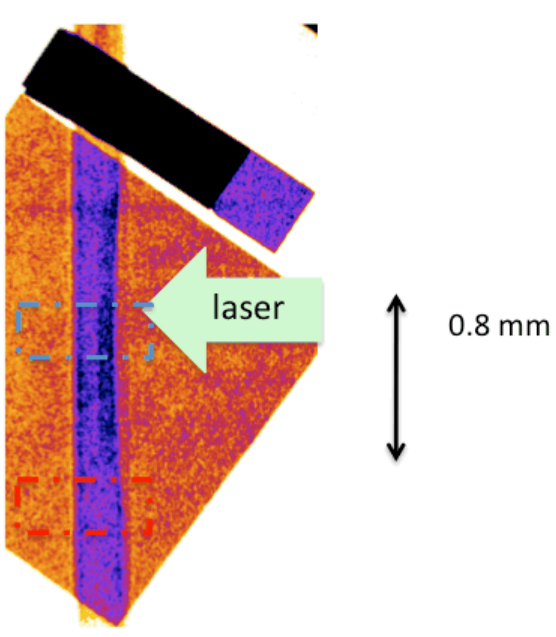

figure 5 


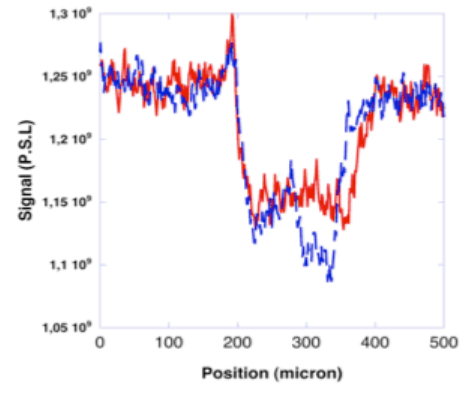

a

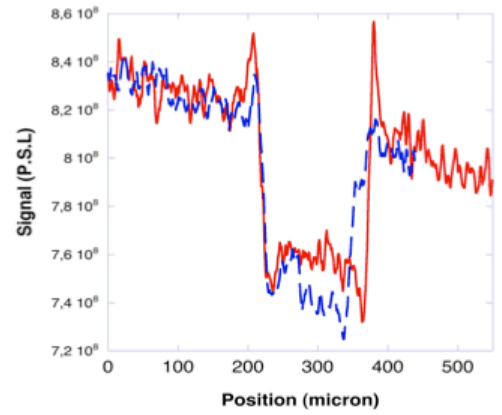

b

figure 6 